PROCEEDINGS OF THE

AMERICAN MATHEMATICAL SOCIETY

Volume 132, Number 9, Pages 2693-2701

S 0002-9939(04)07444-1

Article electronically published on April 21, 2004

\title{
LOGARITHMIC DERIVATIVES OF SOLUTIONS TO LINEAR DIFFERENTIAL EQUATIONS
}

\author{
CHRISTOPHER J. HILLAR
}

(Communicated by Michael Stillman)

\begin{abstract}
Given an ordinary differential field $K$ of characteristic zero, it is known that if $y$ and $1 / y$ satisfy linear differential equations with coefficients in $K$, then $y^{\prime} / y$ is algebraic over $K$. We present a new short proof of this fact using Gröbner basis techniques and give a direct method for finding a polynomial over $K$ that $y^{\prime} / y$ satisfies. Moreover, we provide explicit degree bounds and extend the result to fields with positive characteristic. Finally, we give an application of our method to a class of nonlinear differential equations.
\end{abstract}

\section{INTRODUCTION}

Using Gröbner basis techniques, we provide new constructive proofs of two theorems of Harris and Sibuya [3, 2] (see also, 6, 7] and [8, Problem 6.60]) that give degree bounds and allow for several generalizations. Their results were the following.

Proposition 1.1. Let $N_{1}, N_{2}>1$ be positive integers, and let $K$ be a differential field of characteristic 0 . Let $F$ be a (differential) field extension of $K$, and suppose that $L_{1}(Y)$ and $L_{2}(Y)$ are nonzero homogeneous linear differential polynomials (of orders $N_{1}$ and $N_{2}$ respectively) with coefficients in $K$. Furthermore, suppose that one of the following holds:

(1) $y \in F$ has $L_{1}(y)=L_{2}(1 / y)=0$.

(2) $N_{2} \leq q \in \mathbb{Z}_{+}$, and $y \in F$ has $L_{1}(y)=L_{2}\left(y^{q}\right)=0$.

Then, Dy/y is algebraic over $K$.

In this article, we prove the following more refined result.

Theorem 1.2. Let $N_{1}, N_{2}>1$ be positive integers, and let $K$ be a differential field of characteristic $p$. Let $F$ be a (differential) field extension of $K$. Suppose that $L_{1}(Y)$ and $L_{2}(Y)$ are nonzero homogeneous linear differential polynomials (of orders $N_{1}$ and $N_{2}$ respectively) with coefficients in $K$. Furthermore, suppose that one of the following holds:

(1) $p>N_{1}+N_{2}-2$ and $y \in F$ has $L_{1}(y)=L_{2}(1 / y)=0$.

(2) $p>(q+1)\left(N_{1}-1\right)-1, N_{2} \leq q \in \mathbb{Z}_{+}$, and $y \in F$ has $L_{1}(y)=L_{2}\left(y^{q}\right)=0$.

Received by the editors August 19, 2002 and, in revised form, July 1, 2003.

2000 Mathematics Subject Classification. Primary 34M15, 13P10; Secondary 34A26.

Key words and phrases. Logarithmic derivative, linear differential equation, differential field, Gröbner basis.

This work is supported under a National Science Foundation Graduate Research Fellowship. 
Then, $D^{j} y / y$ is algebraic over $K$ for all $j \geq 1$. Moreover, the degree of the polynomial for $D^{j} y / y\left(j=1, \ldots, N_{1}-1\right)$ in (1) is at most $\frac{\left(N_{2}+N_{1}-2\right) !}{\left(N_{2}-1\right) !}$ and for $(2)$, at most $\frac{\left(q\left(N_{1}-1\right)+N_{1}-2\right) !}{\left(q\left(N_{1}-1\right)-1\right) !}$. Additionally, if $p=0$, then the first condition in (1) and in (2) may be dropped.

This theorem is proved in section 2. In section 3, we give tighter degree bounds under the assumption that $K$ has characteristic zero (or more generally, if $K$ is an infinite perfect field), and finally in section 4 , we describe how our technique applies to certain nonlinear differential equations.

Recall that a polynomial $f \in K[x]$ is called separable if all of its roots are distinct, and a field $K$ is called perfect if every irreducible polynomial in $K[x]$ is separable. Examples of perfect fields include finite fields, fields of characteristic zero, and, of course, algebraically closed fields. It is interesting to note that there is a converse to Theorem 1.2 for perfect fields $K$. Specifically, we have the following result.

Proposition 1.3. Let $K$ be a perfect field. If $y^{\prime} / y$ is algebraic over $K$, then both $y$ and $1 / y$ satisfy linear differential equations over $K$.

Proof. Suppose that $K$ is perfect and that $u=y^{\prime} / y$ is algebraic over $K$. Let $f(x)=x^{m}+a_{m-1} x^{m-1}+\cdots+a_{0} \in K[x]$ be the monic, irreducible polynomial for $u$ over $K$. Since $K$ is perfect, it follows from basic field theory that $\operatorname{gcd}\left(f, \frac{\partial f}{\partial x}\right)=1$. In particular, $\frac{\partial f}{\partial x} \neq 0$. Consider now

$$
0=f(u)^{\prime}=u^{\prime}\left(m u^{m-1}+\sum_{i=1}^{m-1} i a_{i} u^{i-1}\right)+\sum_{i=0}^{m-1} a_{i}^{\prime} u^{i} .
$$

Since $\frac{\partial f}{\partial x}=m x^{m-1}+\sum_{i=1}^{m-1} i a_{i} x^{i-1}$ is not the zero polynomial, it follows that $m u^{m-1}+\sum_{i=1}^{m-1} i a_{i} u^{i-1} \neq 0$ (by irreducibility of $f$ ). Hence $u^{\prime} \in K(u)$, and the same holds for higher derivatives.

Next, notice that $(1 / y)^{\prime}=-y^{\prime} / y^{2}=-u / y$, and an easy induction gives us that $(1 / y)^{(k)}=p_{k}\left(u, u^{\prime}, u^{\prime \prime}, \ldots\right) / y$, in which $p_{k}$ is a polynomial (over $\left.K\right)$ in $u$ and its derivatives (set $\left.p_{0}=1\right)$. By the above, the polynomials $p_{k}\left(u, u^{\prime}, \ldots\right)$ lie in the field $K(u)$. This implies that they satisfy some (nontrivial) linear dependence relation,

$$
\sum_{k=0}^{N} h_{k} p_{k}=0
$$

in which $h_{k} \in K$. Therefore,

$$
0=\sum_{k=0}^{N} h_{k} p_{k} / y=\sum_{k=0}^{N} h_{k}(1 / y)^{(k)},
$$

as desired. Performing a similar examination on the derivatives of $y^{\prime}=u y$ produces a linear differential equation for $y$ over $K$, completing the proof.

As an application of our main theorem, take $F$ to be the field of complex meromorphic functions on $\mathbb{C}$ and $K=\mathbb{Q}$. Then, the only $y$ such that both $y$ and $1 / y$ satisfy linear differential equations over $K$ are the functions $y=c e^{u x}$, in which $u$ is an algebraic number of degree at most $\min \left\{N_{1}, N_{2}\right\}$ and $c \in \mathbb{C} \backslash\{0\}$.

This simple example shows that it is possible to produce a minimum degree of $\min \left\{N_{1}, N_{2}\right\}$ for $y^{\prime} / y$; however, it is still an open question whether we can achieve 
a minimum degree close to the bound given in Theorem 1.2 (or for characteristic zero, Theorem 3.1 below).

Theorem 1.2 can also be used to show that elements in a differential field $F$ do not satisfy linear differential equations over a subfield $K$, as the following example demonstrates.

Example 1.4 (8, Problem 6.59]). Let $K=\mathbb{C}(x)$ and $F=\mathbb{C}((x))$. Then, $\sec (x)$ does not satisfy a linear differential equation over $K$. Indeed, $\cos (x)^{\prime} / \cos (x)=$ $-\tan (x)$ is not algebraic over $\mathbb{C}(x)$.

We begin with a lemma that is fundamental for the solution of this problem, although its generality should be useful in many other contexts. Let $K$ be a field and let $R=K\left[x_{1}, \ldots, x_{n}\right]$ be a polynomial ring over $K$. Give $R$ a grading by assigning to each $x_{i}$ a number $w\left(x_{i}\right)=w_{i} \in \mathbb{N}$ so that

$$
w\left(\prod_{i=1}^{n} x_{i}^{v_{i}}\right)=\sum_{i=1}^{n} v_{i} w_{i} .
$$

Then, we have the following more general version of a theorem of Sperber [7]. Its proof can be found in [5 Lemma 2.2.2].

Lemma 1.5. Let $I$ be the ideal of $R$ generated by a collection of polynomials, and let $J$ be the (homogeneous) ideal generated by the leading forms (with respect to the grading above) of these polynomials. Then, $\operatorname{dim}(J) \geq \operatorname{dim}(I)$.

From this we obtain the immediate corollary that we need.

Corollary 1.6. If $J$ is zero-dimensional, then so is $I$.

\section{The MAin TheOREMS}

Before embarking on proofs of the theorems mentioned in the introduction, we present a simple example to illustrate our technique. Let $y_{1}, y_{2}, \ldots$ be variables. We will view $y_{j}=D^{j} y / y$ as solutions to a system of polynomial equations over $K\left[\left\{y_{j}\right\}_{j=1}^{\infty}\right]$. For example, consider the system $\left(N_{1}=3, N_{2}=2\right)$

$$
\begin{gathered}
y^{\prime \prime \prime}+a_{2} y^{\prime \prime}+a_{1} y^{\prime}+a_{0} y=0, \\
(1 / y)^{\prime \prime}+b_{1}(1 / y)^{\prime}+b_{0}(1 / y)=0,
\end{gathered}
$$

in which $a_{2}, a_{1}, a_{0}, b_{1}, b_{0} \in K$. Dividing the first equation by $y$ and expanding the second one gives us the more suggestive equations

$$
\begin{aligned}
& y_{3}+a_{2} y_{2}+a_{1} y_{1}+a_{0}=0, \\
& \left(2 y_{1}^{2}-y_{2}\right)-b_{1} y_{1}+b_{0}=0 .
\end{aligned}
$$

Also, differentiating the original equation for $1 / y$ and expanding, we have that

$$
-6 y_{1}^{3}+6 y_{1} y_{2}-y_{3}+b_{1}\left(2 y_{1}^{2}-y_{2}\right)-y_{1}\left(b_{1}^{\prime}+b_{0}\right)+b_{0}^{\prime}=0 \text {. }
$$

Thus, we may view $\left(y^{\prime} / y, y^{\prime \prime} / y, y^{\prime \prime \prime} / y\right)=\left(y_{1}, y_{2}, y_{3}\right)$ as a solution to a system of three polynomial equations in three unknowns.

Let $w\left(y_{i}\right)=i$ define a grading of $K\left[y_{1}, y_{2}, y_{3}\right]$, and notice that the system of leading forms, $\left\{y_{3}=0,2 y_{1}^{2}-y_{2}=0,-6 y_{1}^{3}+6 y_{1} y_{2}-y_{3}=0\right\}$, has only the trivial solution $\left(y_{1}, y_{2}, y_{3}\right)=(0,0,0)$. In light of Corollary 1.6, it follows that the equations above define a zero-dimensional variety, establishing Theorem 1.2 (1) for this example. 
In general, we will construct a system of $N_{1}-1$ equations in $N_{1}-1$ unknowns satisfied by the $y_{i}$. These equations will define a zero-dimensional variety, and thus, standard elimination techniques (see, for instance, [1]) give us a direct method of computing, for each $i$, a nonzero polynomial (over $K$ ) satisfied by $y_{i}$.

Let us first examine what happens when we compute $f_{n}=D^{n}(1 / y)$. Notice that

$$
\begin{gathered}
f_{0}=1 / y, \\
f_{1}=-y^{-2} D y=-y_{1} / y, \\
f_{2}=2 y^{-3}(D y)^{2}-y^{-2} D^{2} y=2 y_{1}^{2} / y-y_{2} / y, \\
f_{3}=-6 y_{1}^{3} / y+6 y_{1} y_{2} / y-y_{3} / y .
\end{gathered}
$$

In general, these functions $f_{n}$ can be expressed in the form $f_{n}=(1 / y) p_{n}\left(y_{1}, \ldots, y_{n}\right)$ for polynomials $p_{n} \in \mathbb{Z}\left[y_{1}, \ldots, y_{n}\right]$. More remarkably, with respect to the grading $w\left(y_{i}\right)=i$, these $p_{n}$ are homogeneous of degree $n$. These facts are easily deduced from the following lemma.

Lemma 2.1. Let $m \in \mathbb{Z}_{+}$. Then,

$$
\frac{p_{m}}{m !}=-\sum_{j=1}^{m-1} \frac{p_{m-j}}{(m-j) !} \frac{y_{j}}{j !}-\frac{y_{m}}{m !} .
$$

Proof. Consider the following well-known identity (Leibniz' rule):

$$
\sum_{j=0}^{m}\left(\begin{array}{c}
m \\
j
\end{array}\right)\left(D^{j} h\right)\left(D^{m-j} g\right)=D^{m}(h g) \text {. }
$$

Setting $h=y$ and $g=1 / y$, it follows that

$$
\sum_{j=0}^{m} \frac{D^{j} y}{j !} \frac{D^{m-j}(1 / y)}{(m-j) !}=0 .
$$

Multiplying the numerator and denominator by $y$ and rewriting this expression gives us

$$
\frac{p_{m}}{m !}=-\frac{p_{m-1}}{(m-1) !} \frac{y_{1}}{1 !}-\frac{p_{m-2}}{(m-2) !} \frac{y_{2}}{2 !}-\cdots-\frac{p_{1}}{1 !} \frac{y_{m-1}}{(m-1) !}-\frac{y_{m}}{m !} .
$$

We are now ready to prove Theorem $1.2(1)$.

Proof of Theorem 1.2 (1). With $N_{1}, N_{2}$ as in Theorem 1.2, we suppose $N_{1}=n$, $N_{2}=m$. Dividing through by $y$ in the first differential equation for $y$ gives us

$$
y_{n}=-a_{n-1} y_{n-1}-\cdots-a_{1} y_{1}-a_{0}, \quad a_{i} \in K,
$$

while multiplying the second one for $1 / y$ by $y$ produces the equation

$$
p_{m}+b_{m-1} p_{m-1}+\cdots+b_{0}=0, \quad b_{i} \in K .
$$

Differentiating $k$ times the original linear differential equation for $y$, we will arrive at linear equations $y_{n+k}=L_{k}\left(y_{1}, \ldots, y_{n-1}\right)$ in terms (over $K$ ) of $y_{1}, \ldots, y_{n-1}$ such as (2.1) above (by repeated substitution of the previous linear equations). If we also differentiate $k$ times the equation for $1 / y$, we will produce another equation for the variables $y_{i}$. More formally, we have that

$$
D^{m+k}(1 / y)+D^{k}\left(b_{m-1} D^{m-1}(1 / y)\right)+\cdots+D^{k}\left(b_{0} / y\right)=0
$$


produces the equation (by Leibniz' rule)

$$
D^{m+k}(1 / y)+\sum_{i=0}^{m-1} \sum_{j=0}^{k}\left(\begin{array}{c}
k \\
j
\end{array}\right)\left(D^{j} b_{i}\right)\left(D^{k-j+i}(1 / y)\right)=0 .
$$

So finally (after multiplying through by $y$ ), it follows that

$$
P_{m+k}:=p_{m+k}+\sum_{i=0}^{m-1} \sum_{j=0}^{k}\left(\begin{array}{l}
k \\
j
\end{array}\right)\left(D^{j} b_{i}\right) p_{k-j+i}=0 .
$$

It is clear that the leading homogeneous forms of the $P_{m+k}$ (with respect to the grading above) are $p_{m+k}$. Consider now the ring homomorphism $\phi: K\left[\left\{y_{i}\right\}_{i=1}^{\infty}\right] \rightarrow$ $K\left[y_{1}, \ldots, y_{n-1}\right]$ defined by sending $y_{j} \mapsto 0$ for $j \geq n$ and $y_{j} \mapsto y_{j}$ for $j<n$. Let $\tilde{P}_{m+k}$ denote the polynomials produced by substituting the linear forms $L_{i}$ for the variables $y_{n+i}(i=0,1, \ldots)$ into the polynomials $P_{m+k}$. The leading homogeneous forms of the $\tilde{P}_{m+k}$ will just be $\tilde{p}_{m+k}:=\phi\left(p_{m+k}\right)$, because we are substituting linear polynomials with strictly smaller degree (corresponding to the grading). In light of Corollary 1.6, it suffices to show that the $n-1$ equations (in the $n-1$ variables)

$$
\tilde{p}_{m}=0, \tilde{p}_{m+1}=0, \ldots, \tilde{p}_{m+n-2}=0
$$

are only satisfied by the point $(0, \ldots, 0)$ to prove the claim.

As for the degree bounds in the theorem, simply notice that each polynomial, $\tilde{p}_{m+k}$, has total degree $m+k$ (coming from the $y_{1}^{m+k}$ term), so that Bézout's theorem gives us the bound of $\frac{(m+n-2) !}{(m-1) !}$.

Suppose that $\left(y_{1}, \ldots, y_{n-1}\right) \neq(0, \ldots, 0)$ is a zero of the system in (2.3); we will derive a contradiction. Let $r \in\{1, \ldots, n-1\}$ be the largest integer such that $y_{r} \neq 0$, and choose $t \in\{0, \ldots, m-1\}$ maximal such that $\tilde{p}_{m-t}=0, \tilde{p}_{m-t+1}=0$, $\ldots, \tilde{p}_{m}=0$. If $t=m-1$, then $\tilde{p}_{1}=-y_{1}=0$, and so the recurrence in Lemma 2.1 and (2.3) give us that $y_{i}=0$ for $i \in\{1, \ldots, n-1\}$, a contradiction. Thus, $t \leq m-2$. Using Lemma 2.1 with $\phi$ (and the maximality of $r$ ), examine the following identity: $\frac{\tilde{p}_{m-t+r-1}}{(m-t+r-1) !}=-\frac{\tilde{p}_{m-t+r-2}}{(m-t+r-2) !} \frac{y_{1}}{1 !}-\cdots-\frac{\tilde{p}_{m-t}}{(m-t) !} \frac{y_{r-1}}{(r-1) !}-\frac{\tilde{p}_{m-t-1}}{(m-t-1) !} \frac{y_{r}}{r !}$.

From (2.3) and the property of $t$ above, it follows that $\frac{\tilde{p}_{m-t-1}}{(m-t-1) !} \frac{y_{r}}{r !}=0$. Thus, $y_{r}=0$ or $\tilde{p}_{m-(t+1)}=0$; the first possibility contradicts $y_{r} \neq 0$, while the second contradicts the maximality of $t$.

This proves that the equations (2.3) define a zero-dimensional variety. Finally, we remark that our methods still hold if the characteristic of $K$ is larger than the denominator $(m+n-2)$ above. This completes the proof of the theorem.

The proof for Theorem $1.2(2)$ is similar to the one above, only the recurrences as in Lemma 2.1 are somewhat more complicated. Let $n \in \mathbb{N}, q \in \mathbb{Z}_{+}$ and examine $f_{n, q}=D^{n}\left(y^{q}\right)$. It turns out that $f_{n, q}=y^{q} p_{n, q}\left(y_{1}, \ldots, y_{n}\right)$, in which $p_{n, q} \in \mathbb{Z}\left[y_{1}, \ldots, y_{n}\right]$ is homogeneous of degree $n$ (with respect to the grading $\left.w\left(y_{i}\right)=i\right)$. This follows in a similar manner as before from the following lemma.

Lemma 2.2. Let $p_{n, 1}=y_{n}$ for $n \in \mathbb{N}\left(y_{0}=1\right)$. Then, for all $m \in \mathbb{N}, q>1$,

$$
p_{m, q}=y_{m}+\sum_{j=0}^{m-1}\left(\begin{array}{c}
m \\
j
\end{array}\right) y_{j} p_{m-j, q-1} \text {. }
$$


Proof. Use Leibniz' rule as in Lemma 2.1] with $h=y^{q-1}$ and $g=y$.

The next lemma will be used in the proof of Theorem 1.2(2), and it follows from a straightforward induction on $a$ (using Lemma 2.2).

Lemma 2.3. Let $\phi$ be as in the proof of Theorem 1.2(1) and $n \geq 2$. Then, for all $a \in \mathbb{Z}_{+}$and $b \in \mathbb{N}$, we have $\phi\left(p_{(a+1)(n-1)+b, a}\right)=0$.

We now prove Theorem 1.2 (2).

Proof of Theorem 1.2 (2). With $N_{1}, N_{2}$ as in Theorem 1.2, we suppose $N_{1}=n$, $N_{2}=m \leq q$. As before, the first differential equation for $y$ gives us

$$
y_{n}=-a_{n-1} y_{n-1}-\cdots-a_{1} y_{1}-a_{0}, \quad a_{i} \in K,
$$

while the second one for $y^{q}$ (after dividing through by $y^{q}$ ) produces the equation

$$
p_{m, q}+b_{m-1} p_{m-1, q}+\cdots+b_{0}=0, \quad b_{i} \in K .
$$

Differentiating $k$ times the original linear differential equation for $y$ produces linear equations $y_{n+k}=L_{k}\left(y_{1}, \ldots, y_{n-1}\right)$ in terms (over $K$ ) of $y_{1}, \ldots, y_{n-1}$ such as (2.4) above. If we also differentiate $k$ times the equation for $y^{q}$, we will arrive at another equation for the variables $y_{i}$ :

$$
P_{m+k, q}:=p_{m+k, q}+\sum_{i=0}^{m-1} \sum_{j=0}^{k}\left(\begin{array}{l}
k \\
j
\end{array}\right)\left(D^{j} b_{i}\right) p_{k-j+i, q}=0 .
$$

It is clear that the leading homogeneous forms of the $P_{m+k, q}$ (with respect to the grading above) are $p_{m+k, q}$. Let $\phi$ be as in the proof of Theorem 1.2 (1), and let $\tilde{P}_{m+k, q}$ denote the polynomials produced by substituting the linear forms $L_{i}$ for the variables $y_{n+i}(i=0,1, \ldots)$ into the polynomials $P_{m+k, q}$. If $\tilde{p}_{m+k, q}:=$ $\phi\left(p_{m+k, q}\right) \neq 0$, then the leading homogeneous form of $\tilde{P}_{m+k, q}$ is $\tilde{p}_{m+k, q}$, because we are substituting linear polynomials with strictly smaller degree (corresponding to the grading).

Consider the following system of equations (recall that $q \geq m$ and $n \geq 2$ ):

$$
\tilde{p}_{m, q}=0, \tilde{p}_{m+1, q}=0, \ldots, \tilde{p}_{(q+1)(n-1)-1, q}=0 .
$$

We claim that $(0, \ldots, 0)$ is the only solution to (2.5). Suppose, on the contrary, that $\left(y_{1}, \ldots, y_{n-1}\right) \neq(0, \ldots, 0)$ is a solution to (2.5), and let $r \in\{1, \ldots, n-1\}$ be the largest integer such that $y_{r} \neq 0$. Also, choose $t \in\{1, \ldots, q\}$ minimial such that

$$
\tilde{p}_{t r, t}=0, \tilde{p}_{t r+1, t}=0, \ldots, \tilde{p}_{(t+1) r-1, t}=0 .
$$

Clearly $t \neq 1$, since then $\tilde{p}_{r, 1}=y_{r}=0$, a contradiction. Applying Lemma 2.2 with $\phi$ (and maximality of $r$ ), examine the equation

$$
\tilde{p}_{(t+1) r-1, t}=\tilde{p}_{(t+1) r-1, t-1}+\cdots+\left(\begin{array}{c}
(t+1) r-1 \\
r
\end{array}\right) y_{r} \tilde{p}_{t r-1, t-1} .
$$

Using Lemma 2.3 (with $a=t-1$ ) and the maximality of $r$, we have $\tilde{p}_{t r+b, t-1}=0$ for all $b \in \mathbb{N}$. Consequently, (2.7) and (2.6) imply that $\tilde{p}_{t r-1, t-1}=0$. Repeating this examination with $\tilde{p}_{(t+1) r-2, t}, \tilde{p}_{(t+1) r-3, t}, \ldots, \tilde{p}_{t r, t}$ (in that order) in place of $\tilde{p}_{(t+1) r-1, t}$ on the left-hand side of (2.7), it follows that $\tilde{p}_{t r-i, t-1}=0$ for $i=1, \ldots, r$. This, of course, contradicts the minimality of $t$ and proves the claim. 
It now follows from Corollary [1.6 that the variety determined by

$$
\left\{\tilde{P}_{m, q}=0, \ldots, \tilde{P}_{(q+1)(n-1)-1, q}=0\right\}
$$

is zero-dimensional. Therefore, by an elementary dimension argument, there exists a subset of at most $n-1$ of these equations that generates a zero-dimensional ideal. The degree bounds in the theorem now follow by noticing that each polynomial $\tilde{P}_{m+k, q}$ has total degree at most $m+k$, so that Bézout's theorem gives us the bound of $\frac{(q(n-1)+n-2) !}{(q(n-1)-1) !}$.

Finally, we remark that the methods above still hold if the characteristic of $K$ is larger than $(q+1)(n-1)-1$.

\section{Tighter DEgree Bounds}

It is possible to refine the degree bounds in our main theorem if we restrict our attention to fields of characteristic zero (see Remark 3.2 concerning the perfect field case).

Theorem 3.1. Assuming the hypotheses as in Theorem 1.2 with $K$ having characteristic zero, the degree of the polynomial for $D^{j} y / y\left(j=1, \ldots, N_{1}-1\right)$ over $K$ in (1) is at most $\left(\begin{array}{c}N_{2}+N_{1}-2 \\ N_{1}-1\end{array}\right)$, and for (2), at most $\left(\begin{array}{c}q\left(N_{1}-1\right)+N_{1}-2 \\ N_{1}-1\end{array}\right)$.

Proof. We prove the result for (1), since the other case is the same. Let $N_{1}=n$, $N_{2}=m$ and set $\tilde{P}_{m+k} \in K\left[y_{1}, \ldots, y_{n-1}\right](k=0, \ldots, n-2)$ to be the polynomials in (2.2) after substitution of the linear forms $y_{n+i}=L_{i}\left(y_{1}, \ldots, y_{n-1}\right)$. Corresponding to the grading $w\left(y_{j}\right)=j$, the weight of each monomial in $\tilde{P}_{m+k}$ is less than or equal to $m+k$. Let $\tilde{S}$ be the set of all (affine) solutions with coordinates in $\bar{K}$ to the system $\left\{\tilde{P}_{m+k}=0\right\}_{k=0}^{n-2}$. Our first goal is to bound the cardinality of $\tilde{S}$ by $\left(\begin{array}{c}m+n-2 \\ n-1\end{array}\right)$.

Suppose that $\left\{y_{i, 1}, \ldots, y_{i, s}\right\}$ is the list of all $s$ distinct $i$-th coordinates of members of $\tilde{S}$. Since $K$ is infinite, there exists $k_{i} \in K$ such that $y_{i, j} \neq k_{i}$ for $j=1, \ldots, s$. Now, let $x_{1}, \ldots, x_{n-1}$ be variables and consider the new polynomials $F_{m+k} \in K\left[x_{1}, \ldots, x_{n-1}\right]$ produced by the substitution $y_{i}=x_{i}^{i}+k_{i}$ in the $\tilde{P}_{m+k}$. Since the $n-1$ equations $\tilde{P}_{m+k}=0$ define a zero-dimensional variety, so do the $n-1$ equations $F_{m+k}=0$.

Let $S$ denote the set of all solutions with coordinates in $\bar{K}$ to the system $\left\{F_{m+k}=\right.$ $0\}_{k=0}^{n-2}$. Since the total degree of each $F_{m+k}$ is just $m+k$, we have, by Bézout's theorem,

$$
|S| \leq \frac{(m+n-2) !}{(m-1) !}=(n-1) !\left(\begin{array}{c}
m+n-2 \\
n-1
\end{array}\right) .
$$

Consider the (set-theoretic) map $\psi: S \rightarrow \tilde{S}$ given by

$$
\left(x_{1}, \ldots, x_{n-1}\right) \mapsto\left(x_{1}+k_{1}, \ldots, x_{n-1}^{n-1}+k_{n-1}\right) .
$$

It is easy to see that

$$
\sum_{s \in \tilde{S}}\left|\psi^{-1}(s)\right|=|S|
$$

Let $\left(y_{1}, \ldots, y_{n-1}\right) \in \tilde{S}$. By our choice of $k_{i}$, the polynomial $h_{i}\left(x_{i}\right)=x_{i}^{i}+k_{i}-y_{i}$ has precisely $i$ distinct zeroes. These $i$ roots are distinct, since characteristic zero 
implies that $\operatorname{gcd}\left(h_{i}, \frac{\partial h_{i}}{\partial x_{i}}\right)=1$. Hence, $\left|\psi^{-1}(s)\right| \geq(n-1)$ ! for all $s \in \tilde{S}$, and so from

$$
|\tilde{S}|(n-1) ! \leq|S| \leq\left(\begin{array}{c}
m+n-2 \\
n-1
\end{array}\right)(n-1) !
$$

we arrive at the desired bound on $|\tilde{S}|$.

Finally, suppose that $g_{i}(x) \in K[x]$ is the irreducible polynomial for $y_{i}$ over $K$. Since $K$ has characteristic zero, this polynomial has distinct roots. Thus, there are $\operatorname{deg}\left(g_{i}\right)$ distinct embeddings $\sigma: K\left(y_{i}\right) \rightarrow \bar{K}$ that are the identity on $K$. Moreover, each of these homomorphisms extends to an embedding $\tilde{\sigma}: \bar{K} \rightarrow \bar{K}$ [4, p. 233]. In particular, the $\operatorname{deg}\left(g_{i}\right)$ points, $\left(\tilde{\sigma} y_{1}, \ldots, \tilde{\sigma} y_{n-1}\right)$, are all distinct elements of $\tilde{S}$. Thus, from our bound on $|\tilde{S}|$, we must have

$$
\operatorname{deg}\left(g_{i}\right) \leq|\tilde{S}| \leq\left(\begin{array}{c}
m+n-2 \\
n-1
\end{array}\right)
$$

This completes the proof of the theorem.

Remark 3.2. Examining the proof of Theorem 3.1, it should be clear that the same result holds when $K$ is an infinite perfect field and the hypotheses of Theorem 1.2 are satisfied.

We should also note that the proof above generalizes to bound the number of distinct solutions to certain systems of equations. Specifically, we have the following interesting fact.

Theorem 3.3. Let $w\left(y_{j}\right)=j$ be the grading as above, and let $K$ be a field of characteristic zero. Let $m \in \mathbb{Z}_{+}$and suppose that $\left\{F_{m+k}\left(y_{1}, \ldots, y_{n-1}\right)=0\right\}_{k=0}^{n-2}$ is a zero-dimensional system of polynomial equations over $K$ such that each monomial in $F_{m+k}$ has weight less than or equal to $m+k$. Then, this system will have at most $\left(\begin{array}{c}m+n-2 \\ n-1\end{array}\right)$ distinct solutions with coordinates in $\bar{K}$.

In principle, the number of solutions for a generic system with conditions as in Theorem 3.3 can be found by a mixed volume computation and Bernstein's Theorem (see 1], for instance). This approach, however, seems difficult to implement.

\section{Applications to nOnlinear differential equations}

In the proof of Theorem 1.2 it is clear that the important attributes of the recursions as in (2.1) are that they reduce the degree and are polynomial in nature. In particular, it was not necessary that they be linear. For example, the system

$$
\begin{gathered}
y y^{\prime \prime \prime}+a\left(y^{\prime}\right)^{2}+b y^{2}=0, \\
(1 / y)^{\prime \prime}+c(1 / y)^{\prime}+d(1 / y)=0
\end{gathered}
$$

gives us the recurrence $y_{3}+a y_{1}^{2}+b=0$, which has $y_{3}$ expressible as a polynomial in $y_{1}, y_{2}$ with strictly smaller weight. Repeated differentiation of this equation preserves this property. In general, let $h \in K\left[z_{1}, \ldots, z_{n}\right]$ be a homogeneous polynomial (with respect to total degree) such that each monomial $z^{\alpha}=z_{1}^{\alpha_{1}} \cdots z_{n}^{\alpha_{n}}$ has

$$
\sum_{i=1}^{n}(i-1) \alpha_{i}<n
$$

If the hypotheses of Theorem 1.2 are weakened to allow $y$ to satisfy an equation of the form $D^{n} y=h\left(y, D y, \ldots, D^{(n-1)} y\right)$, then the proof applies without change. A 
generalization along these lines was also considered by Sperber in [7]; however, the techniques developed here give us degree bounds just as in Theorem 1.2 and also allow for a positive characteristic.

\section{ACKNOWLEDGEMENT}

We would like to thank Michael Singer and Bernd Sturmfels for several interesting and useful discussions about this problem.

\section{REFERENCES}

[1] D. Cox, J. Little, and D. O'Shea, Using Algebraic Geometry, Springer-Verlag, New York, 1998. MR 99h:13033

[2] W. A. Harris, Jr. and Y. Sibuya, The n-th Roots of Solutions of Linear Ordinary Differential Equations, Proc. Amer. Math. Soc. 97 (1986), 207-211. MR 87m:12007

[3] W. A. Harris, Jr. and Y. Sibuya, The Reciprocals of Solutions of Linear Ordinary Differential Equations, Adv. in Math. 58 (1985), 119-132. MR 87j:34012

[4] S. Lang, Algebra, 3rd edition, Addison-Wesley Publishing Company, Reading, MA, 1993. MR 33:5416

[5] M. Saito, B. Sturmfels, and N. Takayama, Gröbner Deformations of Hypergeometric Differential Equations, Algorithms and Computation in Mathematics, vol. 6, Springer-Verlag, Berlin, 2000. MR 2001i:13036

[6] M. F. Singer, Algebraic Relations among Solutions of Linear Differential Equations, Trans. Amer. Math. Soc. 295 (1986), 753-763. MR 87f:12015

[7] S. Sperber, Solutions of Differential Equations, Pacific Journal of Mathematics 124 (1986), 249-256. MR 87i:12017

[8] R. Stanley, Enumerative Combinatorics, Vol. 2, Cambridge University Press, Cambridge, UK, 1999. MR 2000k:05026

Department of Mathematics, University of California, Berkeley, California 94720

E-mail address: chillar@math.berkeley.edu 